ONLINE MUTATION REPORT

\title{
Somatic mosaicism is rare in unaffected parents of patients with sporadic tuberous sclerosis
}

\author{
P S Roberts, S Dabora, E A Thiele, D N Franz, S Jozwiak, D J Kwiatkowski
}

J Med Genet 2004;41:e69 (http://www.jmedgenet.com/cgi/content/full/41/5/e69). doi: 10.1136/jmg.2003.014126

$\mathrm{T}$ uberous sclerosis is an autosomal dominant hamartoma syndrome with a prevalence of one in 6000 to one in 10000 births. $^{12}$ Most patients with tuberous sclerosis have hamartomas in the brain, skin, kidneys, and heart; involvement of the lung, gastrointestinal tract, bone, retina, and gingiva is less common but is seen often. During early childhood, tuberous sclerosis presents most commonly with seizures that are caused by involvement of the brain by the hallmark cortical tubers. Mental retardation and a variety of developmental disorders including autism are seen often, and specific neurocognitive defects seem to be associated with involvement of the brain in patients with tuberous sclerosis. ${ }^{3}$

Tuberous sclerosis occurs because of mutations in either of two genes-TSCl or TSC2. ${ }^{4}$ Sporadic cases because of new mutations account for about two thirds of all patients that are seen. ${ }^{6}$ Among families with multiple affected members, about half show linkage to TSC1 on $9 \mathrm{q} 34$ and half to TSC2 on $16 \mathrm{p} 13 .^{7}$ No evidence suggests a third locus. ${ }^{8}$ The TSCl gene consists of 23 exons and the TSC2 gene of 42 exons; mutations are distributed widely throughout both genes. ${ }^{7}$

Mutations in TSC2 are much more common than mutations in TSC1 (4.2:1 ratio); this ratio is higher than that predicted by their relative genomic extents and coding regions. ${ }^{7}$ Many different types of mutation are common in the TSC2 gene, including missense, inframe deletion, and large deletion mutations. In contrast, these three types of mutation are relatively rare in $T S C 1,{ }^{9}$ which possibly contributes to the higher rate of cases because of TSC2 mutations than because of TSC1 mutations.

Mosaicism is well known in many autosomal dominant disorders, particularly relatively common diseases with high sporadic case rates (for example, see reference 10 and its cited references). Generalised somatic and confined gonadal mosaicism have been reported previously in families affected by tuberous sclerosis, which has led to the suggestion that it is a relatively common event. ${ }^{11-16}$ We studied a defined cohort of patients with tuberous sclerosis with mutations in TSC1 and TSC2 to explore systematically the occurrence of mosaicism among the parents of sporadic cases of tuberous sclerosis.

\section{METHODS}

We studied patients with tuberous sclerosis and families derived from a collection of 36 American patients obtained through patient derived requests and 165, 61, and 188 patients from academic paediatric neurology practices in Warsaw, Boston, and Cincinnati, respectively. We also studied two previously reported families with two affected children but unaffected parents. ${ }^{14}$ Details on the informed consent process and clinical information collection have been published..$^{17}$ The parents in these families were not studied in detail for evidence of tuberous sclerosis, but they were not felt to be affected according to their children's doctors

We identified mutations in the patients with denaturing high performance liquid chromatography screening of

\section{Key points}

- Tuberous sclerosis is an autosomal dominant hamartoma syndrome caused by mutations in TSC1 or TSC2.

- Previous reports suggested that somatic mosaicism is common in the parents of apparently sporadic cases of tuberous sclerosis.

- Fifty-four parents of 30 patients with sporadic tuberous sclerosis were screened with a highly sensitive, mutation specific amplification technique to search for low level mosaicism.

- The assays used were capable of detecting mosaicism at a level of one in 100 to one in 500 .

- No parents had mosaicism.

- Review of previous cases of somatic mosaicism in tuberous sclerosis indicates that all who were examined carefully met criteria for a definite diagnosis of tuberous sclerosis.

- Somatic mosaicism is rare in parents of patients with sporadic cases of tuberous sclerosis when there is no evidence of tuberous sclerosis.

amplicons of individual exons of TSC1 and TSC2 as described. ${ }^{17}$ We subjected amplicons with elution shifts according to denaturing high performance liquid chromatography to bidirectional sequencing with ABI 377, 3100, or 3730 DNA Sequencers (PE Biosystems, Warrington, UK) with Big Dye terminator chemistries (PE Biosystems, Warrington, UK). We analysed sequence traces with the Gap4 program of the Staden package (www.staden.sourceforge.net), and we positioned sequence variations within TSC1 and TSC2 with Variation Wizard (Mary Pat Reeve Arlington, USA). ${ }^{17}$ We identified all of the mutations in these patients by sequencing without cloning; the signal ratio of mutant to wild type sequence was close to $1: 1$, which suggests that the patients were not mosaics.

For mosaicism analysis of all mutants except the $18 \mathrm{bp}$ deletion (table 1), we designed primers that would allow specific amplification of mutant but not wild type sequences, following established methods. ${ }^{10}{ }^{18}$ We designed mutant specific primers with Primer Express (version 1.5; Applied Biosystems, Foster City, CA, USA), and we oriented them in the forward or reverse directions. The last $3^{\prime}$ base of the primer matched the mutant sequence and the third from last base was changed to be a mismatch with the wildtype and mutant sequences. This combination of changes was necessary to suppress amplification from the wild type allele. The other primer used in the PCR reaction was the same as that used for mutational screening and was labelled with 6FAM. ${ }^{17}$ For each mutation specific primer, we performed a 
temperature titration on an MJR Thermocycler (MJ Research, Cambridge, MA, USA) across a gradient from $55^{\circ}$ to $65^{\circ} \mathrm{C}$. After agarose gel assay, we chose as the annealing temperature the temperature at which the mutation bearing proband amplified but the control DNA did not. In several cases, amplification of control DNA samples was seen at $65^{\circ} \mathrm{C}$ and the temperature titration was extended to $72^{\circ} \mathrm{C}$ in order to find a suitable annealing temperature. In several cases, discrimination of mutant versus wild type sequence could not be achieved, and the primer sequence was changed at the third base position to improve specificity.

We determined the concentration of each DNA sample studied by quantitative polymerase chain reaction (PCR) with 6-FAM labelled primers to amplify exon 26 of the TSC2 gene, as described. ${ }^{17}$ After we normalised DNA concentrations, mosaicism analysis was performed. We analysed proband, parental, and control DNA, three or more dilutions of the proband DNA into water ( 1 to 10,1 to 100 , and 1 to $500)$, similar dilutions into a control DNA sample, and a water control. We performed PCR with AmpliTaq Gold (PE Biosystems, Warrington, UK) in $20 \mu \mathrm{l}$ reactions for 35 cycles and analysed it on an ABI 3100 DNA Sequencer (Applied Biosystems, Foster City, CA, USA) with HD 400 as a size standard. We used Genotyper software (version 3.7; ABI Biosystems, Foster City, CA, USA) to determine the size of the amplicons and to quantify the relative amount of PCR product as the area. Every reaction set was performed in duplicate on separate days to confirm the results.

When mutational screening of a TSC1 or TSC2 amplicon by denaturing high performance liquid chromatography indicated the presence of an elution shift but no sequence variant could be identified by sequence analysis, we cloned the amplicon to permit analysis for mosaicism. We cloned amplicons in the pEF6/V5-His TOPO TA vector (Invitrogen, Carlsbad, CA, USA). We picked 100 bacterial clones and amplified them to identify those that contained a copy of the amplicon. We performed heteroduplex and denaturing high performance liquid chromatography analysis with a control clone to permit identification of those clones likely to contain mutation bearing amplicons. In this way, a patient was identified in whom the TSC2 mutation del3206-3207 was present in two of 93 clones. This patient served as a positive control for the mosaicism detection strategy described above.

The TSC2 E40 18 bp deletion mutation was analysed by a different technique because of the presence of a repeat sequence that flanked the deletion (table 1). After DNA concentration normalisation, we prepared a series of DNA samples at identical concentrations, including proband DNA diluted into a control DNA sample at ratios of 1:10, 1:25, 1:50, and 1:100. We performed PCR amplification of exon 40, including one primer labelled with 6-FAM, as above, with subsequent analysis on the ABI 3100 DNA Sequencer (PE Biosystems, Warrington, UK). We used Genotyper (version 3.7; ABI Biosystems, Foster City, CA, USA) to quantify the amount of product that corresponded to the deleted, mutant allele for comparison among the different samples analysed. We performed duplicate analyses on different days for each DNA sample.

\section{RESULTS}

Screening for somatic mosaicism was performed in 30 families that contained an apparent sporadic case of

Table 1 Mutations and primer sequences in patients with tuberous sclerosis

\begin{tabular}{|c|c|c|c|c|c|c|c|c|c|c|}
\hline \multicolumn{2}{|c|}{ Patient } & $\begin{array}{l}\text { Number } \\
\text { of } \\
\text { parents } \\
\text { studied }\end{array}$ & Gene & Exon & Mutation & AA effect & Type & Primer sequence & $\begin{array}{l}\text { Anealing } \\
\text { temperature } \\
\left({ }^{\circ}\right)\end{array}$ & $\begin{array}{l}\text { Assay } \\
\text { sensitivity }\end{array}$ \\
\hline 1 & BHM3901 & 2 & TSC1 & 8 & $954 \mathrm{C}>\mathrm{T}$ & $245 R>X$ & Nonsense & GGACCATGAACTGGACCATT & 61 & $<1: 100$ \\
\hline 2 & BG401 & 2 & TSC1 & 8 & $954 \mathrm{C}>\mathrm{T}$ & $245 R>X$ & Nonsense & GGACCATGAACTGGACCATT & 61 & $<1: 200$ \\
\hline 3 & BG4501 & 2 & TSC2 & 7 & 767 ins $G$ & Truncation & Insertion & AAACCCCACCTTCCAGCC & 69 & $<1: 500$ \\
\hline 4 & BG3101 & 2 & TSC2 & 11 & $1219 T>G$ & $407 Y>D$ & Missense & САTCTCTCCACCAGTTCAAATTC & 61 & $<1: 100$ \\
\hline 5 & BHM4701 & 1 & TSC2 & 13 & $1372 \mathrm{C}>\mathrm{T}$ & $458 R>X$ & Nonsense & CCCGCAGGAGCGAGTTCT & 58 & $<1: 500$ \\
\hline 6 & BHM5701 & 2 & TSC2 & 13 & $1372 \mathrm{C}>\mathrm{T}$ & $458 R>X$ & Nonsense & CCCGCAGGAGCGAGTTCT & 58 & $<1: 500$ \\
\hline 7 & BHM1001 & 2 & TSC2 & 13 & $1372 \mathrm{C}>\mathrm{T}$ & $458 R>X$ & Nonsense & CCCGCAGGAGCGAGTTCT & 58 & $<1: 500$ \\
\hline 8 & BHM4401 & 2 & TSC2 & 14 & $1472 \mathrm{C}>\mathrm{A}$ & $491 S>X$ & Nonsense & GGATGTGGGAGAGCTTCT & 58 & $<1: 500$ \\
\hline 9 & $\mathrm{SN}^{*}$ & 1 & TSC2 & 16 & $1831 \mathrm{C}>\mathrm{T}$ & $611 R>W$ & Missense & AGCCACCATACCTGCAGTCA & 66 & $<1: 500$ \\
\hline 10 & BHM3201 & 2 & TSC2 & 16 & $1832 \mathrm{G}>\mathrm{A}$ & $611 R>Q$ & Missense & AGCCACCATACCTGCATCT & 59 & $<1: 500$ \\
\hline 11 & BHM4301 & 2 & TSC2 & 16 & $1832 \mathrm{G}>\mathrm{A}$ & $611 R>Q$ & Missense & AGCCACCATACCTGCATCT & 59 & $<1: 200$ \\
\hline 12 & BHM9701 & 2 & TSC2 & 16 & $1832 \mathrm{G}>\mathrm{A}$ & $611 R>Q$ & Missense & AGCCACCATACCTGCATCT & 59 & $<1: 200$ \\
\hline 13 & BG4001 & 2 & TSC2 & 16 & $1832 \mathrm{G}>\mathrm{A}$ & $611 R>Q$ & Missense & AGCCACCATACCTGCATCT & 59 & $<1: 200$ \\
\hline 14 & BG5301 & 1 & TSC2 & 16 & $1832 G>A$ & $611 R>Q$ & Missense & AGCCACCATACCTGCATCT & 59 & $<1: 200$ \\
\hline 15 & BHM14301 & 2 & TSC2 & 16 & $1832 \mathrm{G}>\mathrm{A}$ & $611 R>Q$ & Missense & AGCCACCATACCTGCATCT & 59 & $<1: 200$ \\
\hline 16 & LR83 & 2 & TSC2 & 29 & $3412 \mathrm{C}>\mathrm{T}$ & $1138 R>X$ & Nonsense & TTCAGGGGGCCATGGTCATT & 57 & $<1: 500$ \\
\hline 17 & BHM401 & 2 & TSC2 & 29 & $3412 \mathrm{C}>\mathrm{T}$ & $1138 R>X$ & Nonsense & TTCAGGGGGCCATGGTCATT & 58 & $<1: 200$ \\
\hline 18 & BHM5801 & 2 & TSC2 & 29 & $3412 \mathrm{C}>\mathrm{T}$ & $1138 R>X$ & Nonsense & TTCAGGGGGCCATGGTCATT & 59 & $<1: 200$ \\
\hline 19 & BHMl4901 & 2 & TSC2 & 29 & $3442 \mathrm{C}>\mathrm{T}$ & $1148 Q>X$ & Nonsense & TTCAGGGGGCCATGGTCATT & 69 & $<1: 100$ \\
\hline 20 & $28-02 \dagger$ & 2 & TSC2 & 33 & $4255 \mathrm{C}>\mathrm{T}$ & $1419 Q>X$ & Nonsense & CCCGTCCAGGGTCCCTGATTA & 69 & $<1: 100$ \\
\hline 21 & BHM12401 & 2 & TSC2 & 33 & $4313 \mathrm{G}>\mathrm{A}$ & $1438 R>Q$ & Missense & GGACCCTCGGGCTGGCTCT & 69 & $<1: 100$ \\
\hline 22 & BHM8201† & 2 & TSC2 & 35 & $4620 \mathrm{C}>\mathrm{A}$ & $1540 Y>X$ & Nonsense & CGGCGATCTTGTGGGTGACT & 64 & $<1: 500$ \\
\hline 23 & LR85 & 2 & TSC2 & 38 & $5024 \mathrm{C}>\mathrm{T}$ & $1675 P>L$ & Missense & TCCACGTGATCGTCACTCT & 65 & $<1: 100$ \\
\hline 24 & BHM2601 & 1 & TSC2 & 38 & $5024 \mathrm{C}>\mathrm{T}$ & $1675 \mathrm{P}>\mathrm{L}$ & Missense & TCCACGTGATCGTCACTCT & 65 & $<1: 100$ \\
\hline 25 & BHM2601 & 1 & TSC2 & 38 & $5024 \mathrm{C}>\mathrm{T}$ & $1675 \mathrm{P}>\mathrm{L}$ & Missense & TCCACGTGATCGTCACTCT & 65 & $<1: 100$ \\
\hline 26 & BНM9001 & 1 & TSC2 & 38 & $5024 \mathrm{C}>\mathrm{T}$ & $1675 P>L$ & Missense & TCCACGTGATCGTCACTCT & 65 & $<1: 100$ \\
\hline 27 & BHM9201 & 2 & TSC2 & 39 & 5075-8 delAGGG & Truncation & Deletion & AGTCTCCCCAGACATGGCCT & 65 & $<1: 200$ \\
\hline 28 & LR87 & 2 & TSC2 & 40 & $5238-5255$ del 18 & 1746delHIKRLR & $\begin{array}{l}\text { Inframe } \\
\text { deletion }\end{array}$ & & 57 & $<1: 100$ \\
\hline 29 & ONK110 & 2 & TSC2 & 40 & $5238-5255$ del 18 & 1746delHIKRLR & $\begin{array}{l}\text { Inframe } \\
\text { deletion }\end{array}$ & & 57 & $<1: 100$ \\
\hline 30 & ONK114 & 2 & TSC2 & 40 & $5238-5255$ del 18 & 1746delHIKRLR & $\begin{array}{l}\text { Inframe } \\
\text { deletion }\end{array}$ & & 57 & $<1: 100$ \\
\hline 31 & ONK146‡ & 0 & TSC2 & 27 & 3206-7 delTG & Truncation & Deletion & TGGGAACAAGCTTGTCACTGA & 66 & NA \\
\hline
\end{tabular}

*Family with possible gonadal mosaicism in which two half siblings had tuberous sclerosis, so only one parent was a suspect for having somatic mosaicism. †Families with possible gonadal mosaicism, in which two siblings had tuberous sclerosis.

$¥$ Sporadic case in which clone determined rate of mosaicism was $4.3 \%$. 
tuberous sclerosis. We selected 28 families from a set of 450 families affected by tuberous sclerosis in whom we performed TSC1 and TSC2 mutational analysis. Two families had been studied in a previous report. ${ }^{14}$ We chose to include cases in this study on the basis of the availability of parental DNA samples and where possible where the same mutation occurred in more than one family affected by sporadic tuberous sclerosis to enhance the use of each assay developed. Of the 28 families, 17 were from Ohio, five from Massachusetts, three from Poland, and three from other regions of the United States.

For mosaicism analysis, we studied leukocyte DNA from the parents of cases of sporadic tuberous sclerosis. In 27 families with sporadic cases of tuberous sclerosis, we performed analysis of mosaicism with sensitive, allele specific PCR to detect the presence of the sequence alteration. The mutations in these families consisted of seven CpG transition mutations, two non-CpG transitions, three transversions, one insertion of one base pair, and one deletion of four base pairs, with up to six different sporadic patients having the same mutation (table 1). In three of these 27 families, two children had tuberous sclerosis but disease was not evident in the parents.

In each mosaicism assay, we amplified serial dilutions of patient DNA into water and into control DNA samples as controls, and we compared these with PCR products derived from parental and control DNA samples. No evidence for mosaicism was seen in any of the parents of these 27 cases, as parental and control DNA samples gave similar, weak PCR products. The level of sensitivity of this analysis varied somewhat from mutation to mutation, but mosaicism could be excluded in all cases at the level of $1 \%$ and, in some cases, the assay was sensitive enough to exclude mosaicism at a level of $0.2 \%$ (table 1 ). Representative assays and controls are shown in figure 1.

Despite the positive controls used in these assays, we were concerned that the absence of positive findings could represent some type of artefact or experimental error. An additional mutation specific assay thus was developed for a patient with tuberous sclerosis in whom a TSC2 mutation was identified by cloning of alleles at a frequency of 2 in 93 clones (4.3\% mosaicism) (bottom of table 1). A mutation specific primer was synthesised; its sensitivity was tested with the cloned amplicon DNA. This technique detected the mutant sequence more easily in patient's DNA than in multiple control DNA samples (fig 1C).

We also studied three patients who had the same 18 base pair deletion in TSC2 with a different technique, as no mutation specific sequence was available. The analysis for mosaicism relied on the ability to discriminate the presence of the smaller size amplicon because of the deletion. Control samples showed that this technique was sensitive to the presence of mosaicism at a level of $1 \%$ (fig 1 ). No evidence of mosaicism above the level of $1 \%$ was seen in any of six parents we studied.

\section{DISCUSSION}

We studied 30 families affected by sporadic tuberous sclerosis, including three families with two affected children. We found no evidence for somatic mosaicism on the basis of analysis of leukocyte DNA with a minimum sensitivity of $1 \%$ mosaicism.

Somatic mosaicism occurs when mutations develop at a developmental stage past that of the fertilised egg. Through the early blastocyst stage, relatively few cells are destined to form the embryonic body, so a period of several days after fertilisation exists during which mutation in a single cell could lead to significant levels of mosaicism. Analysis of a variety of human genetic disorders has shown that somatic mosaicism may be present in up to $20 \%$ of parents of children with sporadic cases of disease. ${ }^{10}{ }^{19}$ The frequency reported in this literature, however, may reflect underreporting because of the technical challenge of detecting low levels of mosaicism. On the other hand, a tendency toward overreporting of mosaicism in human sporadic genetic disease is possible because of a positive reporting bias.

In tuberous sclerosis, limited previous reports have looked at the occurrence of mosaicism in the parents of sporadic cases of tuberous sclerosis. Ten families in which more than one child with tuberous sclerosis was born to parents without evidence of the disease have been reported. ${ }^{11}{ }^{1420}$ In one report of seven families, molecular studies found a mutation in TSC2 in five cases and in TSC1 in one case. Parental analysis showed no evidence for the mutation in these six families, which suggests the occurrence of confined gonadal (without generalised somatic) mosaicism.

Several reports have characterised the occurrence of somatic mosaicism in patients with tuberous sclerosis. Sampson et al. reported the finding of somatic mosaicism in $7 / 27(26 \%)$ families with combined tuberous sclerosis and early onset polycystic kidney disease because of deletions of portions of TSC2 and PKD1. ${ }^{12}$ Four of the seven mosaic individuals in these families had clear diagnostic evidence of tuberous sclerosis, with mosaicism rates of $40-65 \%$. The remaining three mosaic individuals all also seemed to have some diagnostic evidence of tuberous sclerosis (although full details were not given), and those three had mosaicism rates of $15-45 \%{ }^{12}$ Verhoef et al. reported evidence of mosaicism in founders of six families affected by tuberous sclerosis (five somatic and one gonadal). ${ }^{13}$ The mutations in these six families consisted of two small insertions in TSC1, two point mutations in TSC2, and two larger genomic deletions in TSC2. The level of mosaicism was not determined precisely, and all five people with somatic mosaicism met the diagnostic criteria for tuberous sclerosis, although all had normal intelligence and no history of epilepsy. ${ }^{13}$

We and others have also reported that mosaicism for a TSC1 or TSC2 mutation is found among patients with tuberous sclerosis on a routine basis and can be associated with ordinary, relatively severe tuberous sclerosis. ${ }^{15}{ }^{16}$ We reported a patient with tuberous sclerosis with a $30 \%$ mosaicism for a TSC1 mutation (2122delAC) who had relatively severe disease, including brain involvement, seizures, and mental retardation..$^{15}$ Jones et al. described three people with mosaicism for TSC2 mutations at levels of $13 \%$, $15 \%$, and 35\%; all seemed to meet diagnostic criteria for tuberous sclerosis. More recently, during screening of 317 patients with tuberous sclerosis for mutations by denaturing high performance liquid chromatography, we identified nine $(3 \%)$ patients who were mosaic for a mutation in TSC1 or TSC2 at rates between $10 \%$ and $40 \%$ (unpublished observations). Clinical features of these patients seemed to be similar for those in our patient population with tuberous sclerosis as a whole.

How common is mosaicism in tuberous sclerosis therefore? Our detection of no mosaicism with a highly sensitive assay in the parents of 30 sporadic cases of tuberous sclerosis contrasts with published reports. The difference is not significant when our observations are compared with those of Verhoef $(0 / 30 v 6 / 62, p=0.078)$ but is significant compared with those of Sampson $(0 / 30 v 7 / 27, \mathrm{p}=0.003) .{ }^{12}{ }^{13}$ That all people thus far reported with somatic mosaicism for a TSCl or TSC2 mutation have had diagnostic features of tuberous sclerosis if subjected to full clinical and radiographic evaluation, however, is an important distinction. It thus seems, in general, that a person will have clinical features of tuberous sclerosis above a rather low threshold level of mosaicism for a TSC1 or TSC2 mutation. This seems particularly likely in 


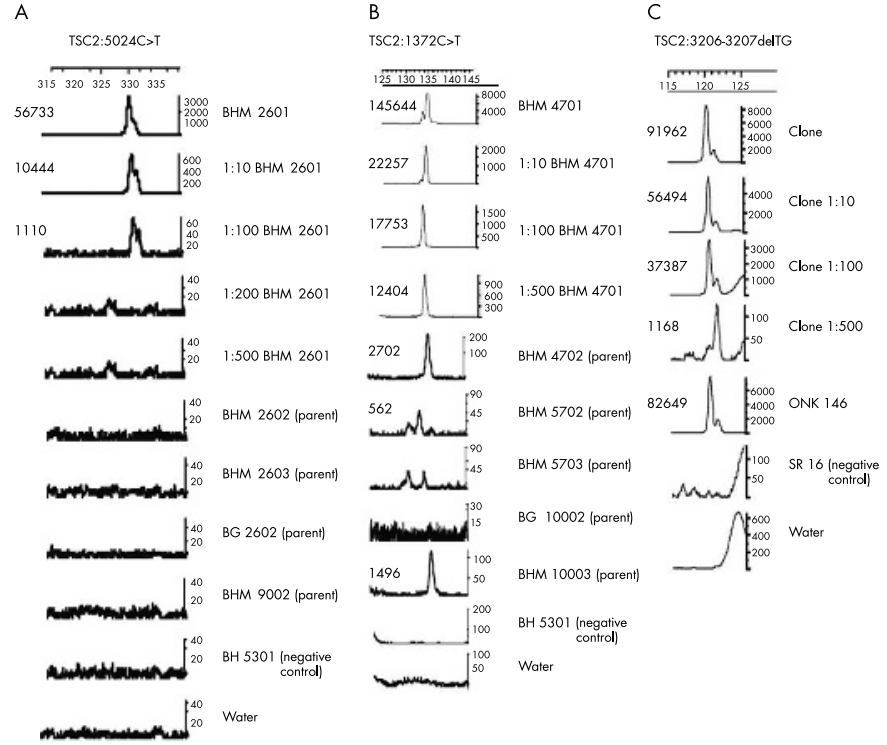

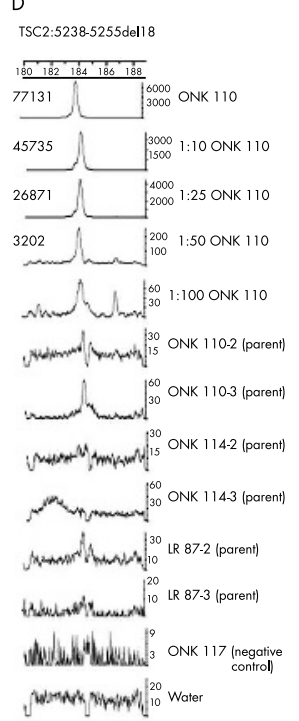

Figure 1 Mosaicism analysis for four different mutations in cases of tuberous sclerosis. Each column shows genotyper output traces of fluorescently labelled PCR products generated with mutation specific primers at varying scales with ABI 3100 DNA Sequencer (Applied Biosystems, Foster City, CA, USA) (small print on right). The "product peak area" is shown to the left of each peak, when of sufficient magnitude. The DNA sample analysed is given at the right of each trace. The figures "1:10", "1:100", etc, indicate the relative dilutions of patient DNA into control DNA (A, B, and D) or into water (C). In all columns, a non-linear decrease is seen in the peak area with the initial dilution because the signal is near the maximum with the undiluted sample. In A and B, the area of the product for each of several parental samples analysed was less than that of the 1:100 (A) or 1:500 (B) dilution of DNA from the patient with the mutation. In C, no control genomic DNA was available to provide dilutions, so cloned DNA was used after quantitation and comparison with patient DNA samples. The patient sample gave a very strong signal comparable with that of the 1:10 dilution of cloned DNA while a negative control DNA sample gave no appreciable signal, like water. In D, only the mutant deletion amplicon product is shown for clarity. All parental samples shown at the bottom had a lower signal than a 1:100 dilution of the mutation bearing sample.

the case of TSC2 mutations, which are associated with more severe clinical manifestations. ${ }^{17}$ This concept also fits well with the observation that a gene mutation associated with tuberous sclerosis will not be identified in $10-15 \%$ of patients with tuberous sclerosis despite comprehensive effort. ${ }^{17}{ }^{21}$ Such patients with tuberous sclerosis, on average, are affected more mildly than typical patients with TSC2 mutations. ${ }^{17}$ Some patients in whom mutations are not identified are likely to be mosaic for a mutation in TSC1 or TSC2 at a relatively low level, making mutation identification difficult or impossible.

Although somatic mosaicism is rare in entirely healthy parents of patients with sporadic tuberous sclerosis, it is seen commonly among patients with sporadic tuberous sclerosis and likely confounds efforts at mutation identification. Furthermore, in the setting of genetic counselling of parents of a child with sporadic tuberous sclerosis, evaluation for subtle but diagnostic evidence for tuberous sclerosis is warranted to help identification of low level somatic mosaicism. Although careful clinical evaluation seems to be an effective screen for somatic mosaicism in tuberous sclerosis, it will not detect gonadal mosaicism. Confined gonadal mosaicism in tuberous sclerosis seems to be relatively rare, however-consistent with the commonly used recurrence risk estimate of $2 \%$ for parents of a child with sporadic tuberous sclerosis. ${ }^{2}$

\section{ACKNOWLEDGEMENTS}

The authors thank the patients and families affected by tuberous sclerosis who participated in these studies and Hope Northrup for contributing DNA samples from one family.

\section{Authors' affiliations}

P S Roberts, S Dabora, D J Kwiatkowski, Hematology Division, Department of Medicine, Brigham and Women's Hospital, Harvard Medical School, Boston, MA 02115, USA

E A Thiele, Department of Pediatrics and Neurology, Massachusetts General Hospital, Boston, MA 02114, USA
D N Franz, Department of Pediatrics and Neurology, Children's Hospital, Cincinnati, OH 45229, USA

S Jozwiak, Department of Child Neurology, Children's Hospital, Warsaw, Poland

Funding: This study was supported by Tuberous Sclerosis Alliance, Rothberg Fund, and NIH NINDS grants 31535 and 24279

Conflicts of interest: none declared.

Correspondence to: Dr Kwiatkowski, Brigham \& Women's Hospital, 221 Longwood Avenue, LMRC 302, Boston, MA 02115, USA; $\mathrm{dk@rics.bwh.harvard.edu}$

Revised version received 4 September 2003

Accepted for publication 28 October 2003

\section{REFERENCES}

1 Osborne JP, Fryer A, Webb D. Epidemiology of tuberous sclerosis. Ann N Y Acad Sci 1991;615: 125-7.

2 Gomez M, Sampson J, Whittemore $V$. The tuberous sclerosis complex. Oxford: Oxford University Press, 1999

3 Bolton PF, Park RJ, Higgins JN, Griffiths PD, Pickles A. Neuro-epileptic determinants of autism spectrum disorders in tuberous sclerosis complex. Brain 2002;125:1247-55.

4 The European Chromosome 16 Tuberous Sclerosis Consortium. Identification and characterization of the tuberous sclerosis gene on chromosome 16. Cell 1993;75:1305-15.

5 Van Slegtenhorst $M$, de Hoogt R, Hermans C, Nellist M, Janssen B, Verhoef S, Lindhout D, van den Ouweland A, Halley D, Young J, Burley M, Jeremiah S, Woodward K, Nahmias J, Fox M, Ekong R, Osborne J, Wolfe J, Povey S, Snell RG, Cheadle JP, Jones AC, Tachataki M, Ravine D, Kwiatkowski DJ. Identification of the tuberous sclerosis gene $\mathrm{TSCl}$ on chromosome $9 \mathrm{q} 34$. Science 1997;277:805-8.

6 Sampson JR, Scahill SJ, Stephenson JB, Mann L, Connor JM. Genetic aspects of tuberous sclerosis in the west of Scotland. J Med Genet 1989:26:28-31.

7 Cheadle JP, Reeve MP, Sampson JR, Kwiatkowski DJ. Molecular genetic advances in tuberous sclerosis. Hum Genet 2000;107:97-114.

8 Povey S, Burley MW, Attwood J, Benham F, Hunt D, Jeremiah SJ, Franklin D, Gillett G, Malas S, Robson EB, Tippett P, Edwards JH, Kwiatkowski DJ, Super M, Mueller R, Fryer A, Clarke A, Webb D, Osborne J. Two loci for tuberous sclerosis: one on $9 q 34$ and one on 16p13. Ann Hum Genet 1994; 58:107-27.

9 Kwiatkowski DJ. Tuberous sclerosis: from tubers to mTOR. Ann Hum Genet 2003;67:87-96. 
10 Leuer M, Oldenburg J, Lavergne JM, Ludwig M, Fregin A, Eigel A, Liung R, Goodeve A, Peake I, Olek K. Somatic mosaicism in hemophilia A: a fairly common event. Am J Hum Genet 2001;69:75-87.

11 Yates JR, van Bakel I, Sepp T, Payne SJ, Webb DW, Nevin NC, Green AJ. Female germline mosaicism in tuberous sclerosis confirmed by molecular genetic analysis. Hum Mol Genet 1997;6:2265-9.

12 Sampson JR, Maheshwar MM, Aspinwall R, Thompson P, Cheadle JP, Ravine D, Roy S, Haan E, Bernstein J, Harris PC. Renal cystic disease in tuberous sclerosis: role of the polycystic kidney disease 1 gene. Am J Hum Genet 1997;61:843-51.

13 Verhoef S, Bakker L, Tempelaars AM, Hesseling-Janssen AL, Mazurczak T, Jozwiak S, Fois A, Bartalini G, Zonnenberg BA, van Essen AJ, Lindhout D, Halley DJ, van den Ouweland AM. High rate of mosaicism in tuberous sclerosis complex. Am J Hum Genet 1999;64:1632-7.

14 Rose VM, Au KS, Pollom G, Roach ES, Prashner HR, Northrup H. Germ-line mosaicism in tuberous sclerosis: how common? Am J Hum Genet 1999;64:986-92.

15 Kwiatkowska J, Wigowska-Sowinska J, Napierala D, Slomski R, Kwiatkowski DJ. Mosaicism in tuberous sclerosis as a potential cause of the failure of molecular diagnosis. N Engl J Med 1999;340:703-7.
16 Jones AC, Sampson JR, Cheadle JP. Low level mosaicism detectable by DHPLC but not by direct sequencing. Hum Mutat 2001;17:233-4.

17 Dabora SL, Jozwiak S, Franz DN, Roberts PS, Nieto A, Chung J, Choy YS, Reeve MP, Thiele E, Egelhoff JC, Kasprzyk-Obara J, Domanska-Pakiela D, Kwiatkowski DJ. Mutational analysis in a cohort of 224 tuberous sclerosis patients indicates increased severity of TSC2, compared with $T S C 1$, disease in multiple organs. Am J Hum Genet 2001;68:64-80.

18 Hezard N, Cornillet P, Droulle C, Gillot L, Potron G, Nguyen P. Factor V Leiden: detection in whole blood by ASA PCR using an additional mismatch in antepenultimate position. Thromb Res 1997;88:59-66.

19 Sippel KC, Fraioli RE, Smith GD, Schalkoff ME, Sutherland J, Gallie BL, Dryja TP. Frequency of somatic and germ-line mosaicism in retinoblastoma: implications for genetic counseling. Am J Hum Genet 1998;62:610-9.

20 Rott HD, Fahsold R. Tuberous sclerosis in two sibs of normal parents. Clin Genet 1991;39:306-8.

21 Jones AC, Shyamsundar MM, Thomas MW, Maynard J, Idziaszczyk S, Tomkins S, Sampson JR, Cheadle JP. Comprehensive mutation analysis of TSC1 and TSC2-and phenotypic correlations in 150 families with tuberous sclerosis. Am J Hum Genet 1999;64:1305-15. 